\title{
Prácticas parentales y conductas internalizantes y externalizadas en niños y niñas de 2 a 5 años
}

\author{
Montserrat Peris Hernández, Gisela Contreras Urrea, \\ Carmen Maganto Mateo y Maite Garaigordobil Landazabal \\ Universidad del País Vasco (España)
}

\begin{abstract}
La etapa preescolar es decisiva para el desarrollo del ser humano por la gran plasticidad que posibilita adquirir nuevas conductas y aprendizajes emocionales. Objetivos: (1) Investigar las conductas externalizadas e internalizadas en niños/niñas preescolares según padres y profesoras, y el involucramiento parental según edad, género y nivel socioeconómico (NSE); (2) Analizar dichas conductas con las prácticas parentales positivas y negativas informadas por padres y educadoras. Participantes: 293 niños y niñas de 2-5 años, 136 niñas (46.4\%) 293 padres y 54 educadoras, de la Concepción, Chile. El diseño es descriptivo, correlacional y de corte trasversal. Evaluación: Child Behavior Check List y Child Teacher Report Form; Parental Sense of Competence; Alabama Parenting Questionnaire-Preschool Revision. Resultados: Los padres evalúan con mayor frecuencia que las educadoras conductas externalizadas e internalizadas. No existen diferencias estadísticamente significativas según edad en comportamientos desadaptativos, mientras que las educadoras evidencian diferencias en conductas externalizadas e internalizadas, presentando los niños más conductas desadaptativas. A menor NSE menos conductas externalizadas e internalizadas perciben padres y educadores, y más prácticas parentales inconsistentes y castigadoras, mientras que a mayor NSE mayor nivel de satisfacción parental. No hay diferencias en satisfacción con el rol parental ni en la percepción de autoeficacia parental.
\end{abstract}

Palabras clave: Conductas externalizadas e internalizadas, prácticas parentales, involucramiento parental, infancia.

Parental practices and behavioral problems. The preschool stage is decisive for the development of the human being, due to the great flexibility that makes it possible to acquire new behaviors and emotional learning. Objectives: (1) To investigate the externalized and internalized behaviors in preschool children according to parents and teachers, and the parental involvement according to age, gender and socioeconomic level (NSE); (2) Analyze these behaviors with the positive and negative parental practices reported by parents and educators. Participants: 293 children aged 2-5 years, 136 girls (46.4\%) 293 parents and 54 teachers, from La Concepción, Chile. The design is descriptive, correlational and cross-sectional. Evaluation: Child Behavior Check List and Child Teacher Report Form; Parental Sense of Competence; Alabama Parenting Questionnaire-Preschool Revision. Results: Parents evaluate more frequently than teachers externalized and internalized behaviors. There are no statistically significant differences according to age in maladaptive behaviors, while educators show differences in externalized and internalized behaviors, with boys presenting more maladaptive behaviors. The lower the NSE, the fewer externalized and internalized behaviors perceived by parents and teachers, and the more inconsistent and punishing parental practices, while the higher the NSE, the higher the level of parental satisfaction. There are no differences in satisfaction with the parental role or in the perception of parental self-efficiency.

Keywords: Externalized and internalized behaviors, parental practices, parental involvement, childhood.

Correspondencia: Carmen Maganto Mateo. Facultad de Psicología. Universidad del País Vasco. Avda. de Tolosa, nº70. C.P.: 20018. San Sebastián (España). E-mail: carmen.maganto@ehu.eus 
Las conductas externalizadas (CExt.) e internalizadas (CInt.) forman parte de la evolución conductual y emocional infantil, pero son también, en sus extremos, los principales motivos de consulta en Salud Mental (Achenbach y Rescorla, 2001; Moreno, Echavarría, Pardo, y Quiñones, 2014).

La presente investigación se organiza en torno a esta distinción. Las CExt. están referidas a problemas de comportamiento que afectan al medio social y a las relaciones con otros. En la niñez toman forma de hiperactividad, agresividad, oposicionismo, comportamiento destructivo, rabietas, desobediencia, impulsividad, falta de atención, actividad motora excesiva, entre otras manifestaciones. Conductas muy propias de los preescolares (Arias, Montoya, y Romero, 2009).

Las CInt. o problemas emocionales en la infancia aparecen en forma de evitación, ansiedad, miedo y depresión. Implican una experiencia interna de inestabilidad emocional, inhibición, timidez, tristeza, soledad, inferioridad, culpa... (López-Soler, Alcántara, Fernández, Castro, y López, 2010).

Los problemas en la niñez son habitualmente identificados por adultos. Una dificultad para establecer la prevalencia de los problemas es que los distintos agentes que conocen al niño/niña valoran de distinta forma sus experiencias y comportamientos, existiendo importantes discrepancias entre los agentes en estas valorizaciones (Maganto y Garaigordobil, 2010).

Durante los primeros años de vida hay muy pocas diferencias de género en los tipos de problemas. Las niñas superan su tendencia a mostrar un comportamiento agresivo y de oposición antes que los niños. Hacia los 4 a 5 años de edad aparecen las diferencias de género en las CExt. (Chaplin, 2015). A partir de esa edad existe una clara tendencia a que los niños presenten más CExt. que las niñas, participando en más actos de agresión y siendo más impulsivos (Cova, Maganto, y Melipillán, 2005). Existe una subestimación en la psicopatología de las niñas, debido a que se evalúa a través de sus adultos significativos (profesores, padres) quienes son más sensibles a los problemas de conducta (Gómez, Santelices, Gómez, Rivera, y Farkas, 2014).

En relación a la edad, las CExt. son identificables desde el segundo año de vida. Con el desarrollo de las capacidades cognitivas y las habilidades para regular las emociones, algunas manifestaciones de agresión física disminuyen con el desarrollo (Contreras, 2016).

Los factores asociados a mayor probabilidad de que un niño/niña presente un problema conductual o emocional están las características personales de los niños/niñas, características de los padres, características de la interacción padre-hijo/hija, factores del contexto familiar, escolares y sociales (Hewitt y Moreno, 2013). Estos factores no tienen una relación determinista sino probabilística y no suelen presentarse de un modo aislado, sino que conforman un verdadero entramado, en el cual el contexto familiar tiene un rol 
fundamental como clave en la génesis y el mantenimiento de las conductas problema (van der Voort et al., 2014).

Las características de los padres y el contexto familiar definen lo que se ha denominado estilo parental. Para Darling y Steinberg (1993) el estilo parental es una constelación de actitudes hacia el niño que en conjunto crean un clima emocional en el cual los comportamientos parentales son expresados. Estas conductas tratan de inculcar en los hijos/as valores, creencias, aspectos de la cultura, habilidades, competencias, etc. (García, Rivera, y Reyes, 2014; Murillo, Priegue, y Cambeiro, 2015). Influyen en el desarrollo socioafectivo, teniendo un rol particularmente determinante en los primeros años de la infancia. Hay PP identificadas como positivas o favorables al desarrollo de habilidades sociales y adaptativas, y en la regulación emocional de niños/as (Gubbins, 2014). Y hay PP negativas por su impacto en el desarrollo infantil y que estarían en la base de problemas emocionales y conductuales (Isaza y Henao, 2012). Las PP más negativas configuran el concepto de maltrato infantil, que implica la privación de alimentación equilibrada, carencia de supervisión, negligencia por falta de dar atención médica necesaria y negligencia escolar (Cabrera, González, y Guevara, 2012).

Muchas conductas disruptivas en los niños están asociadas a PP denominadas inconsistentes por la forma de disciplinar a los hijos. Una parentalidad inconsistente es aquella que reacciona ante la conducta de su hijo, no de acuerdo a la lógica sino de acuerdo a su estado de ánimo, los comportamientos paternos son impredecibles para los hijos, lo que hoy se castiga mañana no y viceversa (Chaplin et al., 2015). Estas prácticas desarrollan problemas con secuelas importantes en el desarrollo futuro, incluso en las relaciones posteriores de amor romántico (Tani y Smorti, 2013).

El desarrollo de comportamientos desadaptativos está influido por una diversidad de aspectos, dentro de los cuales las PP tienen especial relevancia, y en concreto las PP desadaptativas (García et al., 2014), ya que ofrecen un estilo parental autoritario, hostil y castigador (Bolívar et al., 2014).

En familias de niños con CExt. aparecen altos niveles de afecto negativo de forma recíproca (Franco, Pérez, y de Dios, 2014; Moreno et al., 2014).

Atendiendo a estos supuestos, los objetivos del estudio fueron: (1) Describir la percepción de padres y educadoras en CExt y CInt en niños/niñas, así como las prácticas parentales positivas (PP/positivas), inconsistentes (PP/Inconsistentes) y castigadoras (PP/Castigadoras; (2) Analizar las diferencias entre CExt. y CInt. de padres y educadoras en función del sexo, edad y NSE; (3) Relacionar las prácticas parentales con CExt. y CInt. en preescolares según sexo, edad, y NSE. 


\section{MÉTODO}

\section{Participantes}

Los participantes fueron 293 niños/niñas entre 2-5 años, 136 niñas (46.4\%). Se entrevistó a sus progenitores y/o cuidadores principales (293) y sus educadoras (54). Fueron seleccionados atendiendo a la segmentación social de los establecimientos educativos en Chile: privados, subvencionado y municipales, siendo este un buen indicador indirecto de los diferentes grupos socioeconómicos (Bellei, 2013). Grupos alto y medio alto asisten a colegios privados (12 centros); grupos medios a establecimientos subvencionados (10 centros); la población de niveles socioeconómicos más bajos asiste a establecimientos públicos (8 centros). La configuración final se recoge en la tabla 1.

\begin{tabular}{lcccc}
\multicolumn{6}{l}{ Tabla 1. Distribución por sexo, edad, nivel socioeconómico } \\
\hline & $\begin{array}{c}\text { NSE Alto } \\
n=93\end{array}$ & $\begin{array}{c}\text { NSE Medio } \\
n=101\end{array}$ & $\begin{array}{c}\text { NSE Bajo } \\
n=99\end{array}$ & $\begin{array}{c}\text { Totales } \\
n=293\end{array}$ \\
\hline Niños & 48 & 54 & 55 & 157 \\
\hline Niñas & 45 & 47 & 44 & 136 \\
\hline 2 años & 25 & 26 & 25 & 76 \\
\hline 3 años & 26 & 25 & 24 & 75 \\
\hline 4 años & 24 & 25 & 25 & 74 \\
\hline 5 años & 18 & 25 & 25 & 68 \\
\hline
\end{tabular}

\section{Instrumentos}

Child Behavior Check List (CBCL. Achenbach y Rescorla, 2000)

El CBCL es un heterioinforme para registrar problemas conductuales, emocionales y competencias sociales de los niños/niñas. Los padres o cuidadores responden según tres categorías: $0=$ no es cierto; $1=$ algunas veces; $2=$ muy a menudo. En Chile, la escala total muestra un alpha de Cronbach de .95, la escala de internalización de .88 y la de externalización de .90 (Lecannelier et al., 2014).

Child Teacher Report Form (CTRF. Achenbach y Rescorla, 2000)

El CTRF valora las conductas internalizadas y externalizadas evaluadas por los profesores. El formato de aplicación es el mismo que el CBCL con propiedades psicométricas semejantes. Ambos cuestionarios compendian dos grupos de síndromes, internalizado (reactividad emocional, ansiedad/depresión, quejas somáticas y ensimismamiento) y externalizado (problemas de atención y agresividad).

Alabama Parenting Questionnaire-Preschool Revision (APQPR. Clerkin, Marks, Policaro, y Halperin, 2007)

Instrumento autoadministrado de 32 ítems con respuestas que van desde $0=$ Nunca a 5=Siempre. La adaptación para preescolares (APQ-PR) identificó tres factores: parentalidad positiva, negativa/inconsistente y punitiva/castigadora. El análisis 
confirmatorio constató la estructura de los tres factores propuestos. La consistencia interna fue: .88 para prácticas parentales positivas; .76 prácticas parentales inconsistentes y .68 para prácticas parentales castigadoras, y mostró estabilidad temporal en uno año de seguimiento (correlaciones de $.52, .59$ y .80).

\section{Procedimiento}

El estudio tuvo un diseño descriptivo y correlacional de corte trasversal. Se siguieron los principios éticos del Código de ética profesional chileno (1999) en el que se tuvo presente la confidencialidad de los datos y la voluntariedad de la participación en la investigación, así como los principios éticos aprobados por la Sociedad Británica de Psicología (2006).

Los instrumentos fueron autoadministrados a las educadoras, para los padres, de acuerdo al tipo de centro, fueron autoadministrados o aplicados por evaluador cualificado. Este formato subsanaba el que muchas/os de los/as cuidadores tenían una escolaridad muy baja o analfabetismo por desuso.

\section{RESULTADOS}

Para dar respuesta al primer objetivo, describir la percepción de padres y educadoras en CExt. y CInt. en niños y niñas, así como las prácticas parentales, se llevaron a cabo análisis descriptivos cuyos resultados se presentan en la tabla 2.

Tabla 2. Media y desviación estándar de conductas externalizadas e internalizadas según los padres y

\begin{tabular}{|c|c|c|c|}
\hline \multicolumn{4}{|c|}{ educadoras y prácticas parentales } \\
\hline Escala & $N$ & $M$ & $D E$ \\
\hline \multicolumn{4}{|c|}{ Conductas infantiles según los padres } \\
\hline CExt. & 292 & 0.63 & 0.34 \\
\hline CInt. & 292 & 0.29 & 0.20 \\
\hline \multicolumn{4}{|c|}{ Conductas infantiles según las educadoras } \\
\hline CExt. & 293 & 0.36 & 0.37 \\
\hline CInt. & 293 & 0.23 & 0.21 \\
\hline \multicolumn{4}{|c|}{ Prácticas parentales } \\
\hline Positivas & 289 & 3.61 & 0.35 \\
\hline Inconsistentes & 289 & 1.02 & 0.68 \\
\hline Castigadoras & 289 & 0.75 & 0.50 \\
\hline
\end{tabular}

Se observa mayor nivel de conductas desadaptativas informadas por los padres que por las educadoras. Por orden de frecuencia los padres adoptan prácticas parentales positivas, inconsistentes y castigadoras. Para analizar las diferencias entre CExt. y CInt. de padres y educadoras se llevaron a cabo análisis de comparación de medias, presentando los resultados en la tabla 3 . 
PERIS et al. Prácticas parentales y problemas de conducta

Tabla 3. Descriptivos y puntajes $t$ de las conductas externalizadas e internalizadas según padres y educadoras

\begin{tabular}{cccccc}
\hline & \multicolumn{2}{c}{ Padres } & \multicolumn{2}{c}{ Educadoras } & \multirow{2}{*}{$t(g l)$} \\
\cline { 1 - 5 } & $M$ & $D E$ & $M$ & $D E$ & \\
\hline CExt. & 0.63 & 0.34 & 0.36 & 0.37 & $10.901(291)^{* *}$ \\
\hline CInt. & 0.29 & 0.20 & 0.23 & 0.21 & $3.459(291)^{* * *}$ \\
\hline Nota. ${ }^{*} p \leq .05 ; * * \leq .01 ; * * * \leq .001$. & &
\end{tabular}

Las diferencias entre padres y educadoras son estadísticamente significativas (Tabla 3) valorando los padres con mayor severidad las CExt, y CInt. de sus hijos/hijas.

Los análisis diferenciales en los dos tipos de conductas en niños y en niñas aisladamente, se presentan en la tabla 4.

Tabla 4. Descriptivos y puntajes t en conductas externalizadas e internalizadas según padres y educadoras en niños y niñas

\begin{tabular}{|c|c|c|c|c|c|}
\hline \multicolumn{6}{|c|}{ Niños $n=157$} \\
\hline & \multicolumn{2}{|c|}{ Padres } & \multicolumn{2}{|c|}{ Educadoras } & \multirow{2}{*}{$t(g l)$} \\
\hline & $M$ & $D E$ & $M$ & $D E$ & \\
\hline CExt. & 0.674 & 0.368 & 0.443 & 0.414 & $6.304(155)^{* * * *}$ \\
\hline CInt. & 0.305 & 0.207 & 0.265 & 0.228 & $1.831(155)$ \\
\hline \multicolumn{6}{|c|}{ Niñas $n=136$} \\
\hline CExt. & 0.59 & 0.31 & 0.27 & 0.30 & $9.646(135) * * *$ \\
\hline CInt. & 0.28 & 0.19 & 0.21 & 0.20 & $3.139(135)^{* *}$ \\
\hline
\end{tabular}

Se constatan (Tabla 4) diferencias significativas, informando los padres de mayor nivel de CExt. en los niños que las educadoras. En las niñas, también los padres perciben con mayor intensidad ambos tipos de conductas que las educadoras, siendo las diferencias estadísticamente significativas.

Para analizar el grado de concordancia en la evaluación de conductas desadaptativas entre padres y educadores, se realizaron correlaciones bivariadas de Pearson, cuyo valores fueron $r=.29, p=.01$ en CExt, y $r=.18, p=.01$ en CInt.

Con la finalidad de dar respuesta al segundo objetivo, analizar las diferencias de CExt. Y CInt entre padres y educadoras en función del sexo, edad y NSE, se realizaron análisis de comparación de medias presentados en las tablas 5, 6 y 7.

Tabla 5. Medias, desviaciones típicas y resultados de la prueba t en función del sexo en conductas externalizadas e internalizadas según padres y educadoras

\begin{tabular}{cccccc}
\hline & \multicolumn{2}{c}{ Niños $n=157$} & \multicolumn{2}{c}{ Niñas $n=136$} & \multirow{2}{*}{$t(g l)$} \\
\cline { 1 - 5 } & $M$ & $D E$ & $M$ & $D E$ & \\
\hline CExt./padres & $0.67_{\mathrm{a}}$ & 0.36 & $0.59_{\mathrm{a}}$ & 0.31 & $-1.912(290)$ \\
\hline CInt./padres. & $0.30_{\mathrm{a}}$ & 0.20 & $0.28_{\mathrm{a}}$ & 0.20 & $-1.014(290)$ \\
\hline CExt./educadoras & $0.44_{\mathrm{b}}$ & 0.41 & $0.27_{\mathrm{a}}$ & 0.30 & $-3.943(291)^{* * * *}$ \\
\hline CInt./educadoras & $0.26_{\mathrm{b}}$ & 0.22 & $0.21_{\mathrm{a}}$ & 0.20 & $-2.127(291)^{*}$ \\
\hline
\end{tabular}

Nota. Se indican con las mismas letras superíndice las medias que no difieren entre sí, diferentes letras superíndices indica diferencias entre las medias. ${ }^{*} p \leq .05$; $* * p \leq .01 ; * * * p \leq .001$. 
Los padres (Tabla 5) no observan diferencias en los comportamientos desadaptativos de niños y niñas en este grupo etáreo. Las educadoras sí observan diferencias tanto en CExt. ( $p<.001)$ como CInt. ( $p=0.034)$; en ambos casos, los niños son percibidos con mayor nivel de conductas desadaptativas.

Tabla 6. Medias, desviaciones típicas y resultados del ANOVA en función de la edad en conductas externalizadas e internalizadas según padres y educadoras

\begin{tabular}{|c|c|c|c|c|c|c|c|c|c|}
\hline & \multicolumn{2}{|c|}{2 años $n=76$} & \multicolumn{2}{|c|}{3 años $n=75$} & \multicolumn{2}{|c|}{4 años $n=74$} & \multicolumn{2}{|c|}{5 aก̃os $n=68$} & \multirow{2}{*}{$F(g l)$} \\
\hline & $M$ & $D E$ & $M$ & $D E$ & $M$ & $D E$ & $M$ & $D E$ & \\
\hline CExt./padres. & $0.64_{a}$ & 0.33 & $0.63_{\mathrm{a}}$ & 0.31 & $0.65_{\mathrm{a}}$ & 0.36 & $0.60_{\mathrm{a}}$ & 0.36 & $0.231(3.288)$ \\
\hline CInt./padres. & $0.46_{\mathrm{a}}$ & 0.37 & $0.40_{\mathrm{ac}}$ & 0.42 & $0.24 \mathrm{~b}$ & 0.32 & $0.32_{\mathrm{cb}}$ & 0.33 & $5.243(3.289))^{* * *}$ \\
\hline CExt./educadoras. & $0.46_{a}$ & 0.37 & $0.40_{\mathrm{ac}}$ & 0.42 & $0.24_{b}$ & 0.32 & $0.32_{\mathrm{cb}}$ & 0.33 & $5.243(3.289) * *$ \\
\hline CInt./educadoras. & $0.27 \mathrm{a}$ & 0.21 & $0.26_{\mathrm{ac}}$ & 0.22 & $0.16_{\mathrm{b}}$ & 0.18 & $0.21_{\mathrm{cb}}$ & 0.22 & $5.224(3.289) * *$ \\
\hline
\end{tabular}

Nota. Se indican con las mismas letras superíndice las medias que no difieren entre sí, diferentes letras superíndices indica diferencias entre las medias. $* p \leq .05 ; * * p \leq .01 ; * * * p \leq .001$.

De acuerdo al informe de los padres (Tabla 6), no existen diferencias de edad en comportamientos externalizados, pero sí en internalizados, observándose una tendencia decreciente estadísticamente significativa. Según las educadoras, se observan diferencias significativas en CExt. y CInt., con tendencia a disminuir dichas conductas con la edad, aunque no es un descenso lineal, siendo evidentes las diferencias entre 2 y 4 años $(p<0.01)$ y entre 3 y 4 años $(p=0.04)$.

Para analizar las diferencias por NSE en las CExt. y CInt. se realizaron análisis de varianza (Tabla 7).

Tabla 7. Medias, desviaciones típicas y resultados del ANOVA según NSE en conductas externalizadas e internalizadas según padres y educadoras

\begin{tabular}{cccccccc}
\hline & \multicolumn{2}{c}{ NSE Bajo $n=99$} & \multicolumn{2}{c}{ NSE Medio $n=101$} & \multicolumn{2}{c}{ NSE Alto $n=93$} & \multirow{2}{*}{$F(\mathrm{gl})$} \\
\hline & $M$ & $D E$ & $M$ & $D E$ & $M$ & $D E$ & $6.655(2.289)^{* *}$ \\
\hline CExt./padres & $0.72 \mathrm{a}$ & 0.38 & $0.64 \mathrm{a}$ & 0.34 & $0.54 \mathrm{~b}$ & 0.27 & $31.372(2.289)^{* * *}$ \\
\hline CInt./padres. & $0.40 \mathrm{a}$ & 0.24 & $0.28 \mathrm{~b}$ & 0.16 & $0.19 \mathrm{c}$ & 0.13 & $3.990(2.90)^{*}$ \\
\hline CExt./educadoras. & $0.44 \mathrm{a}$ & 0.43 & $0.32 \mathrm{a}$ & 0.33 & $0.30 \mathrm{~b}$ & 0.33 & $6.873(2.90)^{* *}$ \\
\hline CInt./educadoras. & $0.29 \mathrm{a}$ & 0.24 & $0.18 \mathrm{~b}$ & 0.17 & $0.23 \mathrm{a}$ & 0.21 & 6
\end{tabular}

Nota. Se indican con las mismas letras superíndice las medias que no difieren entre sí, diferentes letras superíndices indica diferencias entre las medias. $* p \leq .05 ; * * p \leq .01 ; * * * p \leq .001$.

Existe una clara relación entre NSE y CExt. y CInt. (Tabla 7), tanto si informan los padres como las educadoras. Según los padres, a mayor NSE menor proporción de conductas disfuncionales. En las educadoras ocurre algo similar en CExt., pero en CInt. la tendencia no es tan directa, aunque puede decirse que el NSE bajo presenta más CInt. que el NSE alto.

Respecto al tercer objetivo, relacionar las prácticas parentales positivas y negativas con CExt. y CInt. según sexo, edad, y NSE, los resultados se detallan en las Tablas 8,9 y 10. 
Tabla 8. Medias, desviaciones típicas y resultados de la prueba $t$ en función del sexo según las prácticas parentales de los padres

\begin{tabular}{cccccc}
\hline & \multicolumn{2}{c}{ Niños $n=157$} & \multicolumn{2}{c}{ Niñas $n=136$} & \multirow{2}{*}{$t(g l)$} \\
\cline { 2 - 5 } & $\mathrm{M}$ & $\mathrm{DE}$ & $\mathrm{M}$ & $\mathrm{DE}$ & \\
\hline PP/Positivas & $3.60_{\mathrm{b}}$ & 0.35 & $3.62_{\mathrm{a}}$ & 0.35 & $0.435(287)$ \\
\hline PP/Inconsistentes & $1.09_{\mathrm{b}}$ & 0.70 & $0.94_{\mathrm{a}}$ & 0.65 & $-1.837(287)$ \\
\hline PP/Castigadoras & $0.78_{\mathrm{b}}$ & 0.50 & $0.72_{\mathrm{a}}$ & 0.51 & $-0.974(287)$ \\
\hline
\end{tabular}

Nota. Se indican con las mismas letras superíndice las medias que no difieren entre sí, diferentes letras superíndices indica diferencias entre las medias.

Tal como se ve en la tabla 8 , no se encontraron diferencias estadísticamente significativas en ninguna de las variables.

Tabla 9. Medias, desviaciones típicas y resultados del ANOVA en función de la edad en prácticas parentales

\begin{tabular}{cccccccccc}
\hline & 2 años $n=76$ & \multicolumn{2}{c}{3 años $n=75$} & \multicolumn{2}{c}{4 años $n=74$} & \multicolumn{2}{c}{5 años $n=68$} & \multirow{2}{*}{$F(g l)$} \\
\hline & $\mathrm{M}$ & $\mathrm{DE}$ & $\mathrm{M}$ & $\mathrm{DE}$ & $\mathrm{M}$ & $\mathrm{DE}$ & $\mathrm{M}$ & $\mathrm{DE}$ & \\
\hline PP/Positivas & $3.56_{\mathrm{a}}$ & 0.40 & $3.67_{\mathrm{a}}$ & 0.32 & $3.59_{\mathrm{a}}$ & 0.34 & $3.61_{\mathrm{a}}$ & 0.35 & $1.264(3.285)$ \\
\hline PP/Inconsistentes & $0.98_{\mathrm{a}}$ & 0.67 & $1.01_{\mathrm{a}}$ & 0.69 & $1.00_{\mathrm{a}}$ & 0.66 & $1.08_{\mathrm{a}}$ & 0.71 & $0.304(3.285)$ \\
\hline PP/Castigadoras & $0.61_{\mathrm{a}}$ & 0.43 & $0.68 \mathrm{ac}$ & 0.43 & $0.89 \mathrm{~b}$ & 0.45 & $0.84_{\mathrm{bc}}$ & 0.63 & $5.212(3.285)^{*}$ \\
\hline
\end{tabular}

Nota. Se indican con las mismas letras superíndice las medias que no difieren entre sí, diferentes letras superíndices indica diferencias entre las medias. $* p \leq .05 ; * * p \leq .01 ; * * * p \leq .001$.

La única variable que presenta diferencias estadísticamente significativas son las PP/Castigadoras (Tabla 9). A medida que se incrementa la edad aumentan correlativamente las prácticas castigadoras, encontrándose las mayores diferencias de medias entre 3 y 4 años.

Tabla 10. Medias, desviaciones típicas y resultados del ANOVA en función del NSE en prácticas parentales

\begin{tabular}{cccccccc}
\hline & \multicolumn{2}{c}{ NSE Bajo $n=99$} & \multicolumn{2}{c}{ NSE Medio $n=101$} & \multicolumn{2}{c}{ NSE Alto $n=93$} & $F(g l)$ \\
\cline { 2 - 9 } & $\mathrm{M}$ & $\mathrm{DE}$ & $\mathrm{M}$ & $\mathrm{DE}$ & $\mathrm{M}$ & $\mathrm{DE}$ & \\
\hline PP/Positivas & $3.62_{\mathrm{a}}$ & 0.37 & $3.61_{\mathrm{a}}$ & 0.33 & $3.59_{\mathrm{a}}$ & 0.35 & $0.145(2.86)$ \\
\hline PP/Inconsistentes & $1.25_{\mathrm{a}}$ & 0.80 & $0.94_{\mathrm{b}}$ & 0.55 & $0.84_{\mathrm{b}} \mathrm{c}$ & 0.59 & $10.057(2.286)^{* * * *}$ \\
\hline PP/Castigadoras & $0.90_{\mathrm{a}}$ & 0.56 & $0.73_{\mathrm{b}}$ & 0.43 & $0.62_{\mathrm{bc}}$ & 0.46 & $7.922(2.286)^{* * *}$ \\
\hline
\end{tabular}

Nota. Se indican con las mismas letras superíndice las medias que no difieren entre sí, diferentes letras superíndices indica diferencias entre las medias. ${ }^{*} p \leq .05 ; * * p \leq .01 ; * * * \leq .001$.

Como se ve en la tabla 10 , se encuentran diferencias estadísticamente significativas en las prácticas PP/Inconsistentes y PP/Castigadoras. Las mayores diferencias se aprecian entre los participantes de NSE bajo y medio $(p=0.03)$, y entre los del NSE bajo y alto $(p<0.001)$.

En las PP/Castigadoras las diferencias de medias más significativas fueron encontradas entre el NSE bajo y alto $(p<0.001)$ y entre los del NSE bajo y medio $(p=0.04)$. Se observa una tendencia lineal inversa en función del NSE, donde a menor NSE mayor ejecución de prácticas parentales negativas anteriormente enunciadas. 


\section{DISCUSIÓN Y CONCLUSIONES}

Los resultados que responden al primer objetivo, indican que los padres informan de más CExt. y CInt. que las educadoras, siendo las diferencias estadísticamente significativas. En los chicos, los padres observan más CExt, que las educadoras. En las chicas, en CExt. y CInt. difieren entre sí, informando los padres de mayor nivel de conductas desadaptativas.

Estos resultados van en la línea otros estudios en los que las diferencias entre padres y educadores son evidentes (Maganto y Garaigordobil, 2010). Así mismo se informa de mayor número de CExt. que CInt. en niños que en niñas, por el menor control de su respuesta conductual y por presentar mayor dificultad de respuestas emocionales con sus pares (Cova et al., 2005). Especialmente ocurre con conductas de déficit de atención con hiperactividad, trastorno disocial y oposicionista. También otros estudios constatan más CInt. (depresión, ansiedad, inhibición) en niñas que en niños.

Se podría inferir que existe una asociación género-edad-psicopatología, donde las conductas de tipo externalizantes comenzarían su curso en la infancia y preponderantemente en niños, mientras que las conductas internalizantes aparecían más frecuentemente en niñas. Es posible que haya un sesgo en la evaluación femenina en función de la expectativa de patología y género. De acuerdo con Chaplin et al. (2015) nos inclinamos a pensar que la influencia de los patrones de socialización a los cuales están expuestos los niños/as explica en gran parte estas diferencias.

En el presente estudio, las prácticas parentales con las que los padres educan e interaccionan con sus hijos/as son, por este orden de frecuencia, prácticas parentales positivas, inconsistentes y castigadoras. Estos resultados son confirmados en investigaciones precedentes (Isaza y Henao, 2012). En general, los padres utilizan técnicas positivas en la educación en mayor medida que negativas, excepto en los hogares con adversidad psicosocial, como familias de ambientes desfavorecidos económicamente (Jaramillo, 2015), viviendo una violencia intraparental muy manifiesta (Hewitt y Moreno, 2013) siendo estos factores los desencadenantes o predisponentes de las conductas desadaptativas (van der Voort et al., 2014).

Respecto a los resultados del segundo objetivo, se constata que las diferencias en CExt. y CInt. en función del sexo dependen del informante. Así, por ejemplo, para los padres no hay diferencias en conductas problema entre niños y niñas, mientras que para las educadoras, en ambas conductas hay diferencias significativas con mayor nivel de conductas problema en los niños. Las educadoras, con mayor nivel de observación y conocimiento de las conductas infantiles perciben también diferencias entre niños y niñas en conductas internalizantes. Resultados sobre estas discrepancias han sido ya observadas en investigaciones previas (Gómez et al., 2014). 
Varios autores sugieren que los problemas internalizantes han sido menos estudiados que los problemas conductuales (Moreno et al., 2014) especialmente en preescolares, a quienes no les es fácil comunicar sus emociones de tristeza, soledad, inhibición, temor (Contreras, 2016; López-Soler et al., 2010).

Respecto a la edad, este estudio no ratifica las diferencias en conductas externalizantes según los padres, pero si en conductas internalizantes, obteniendo menor puntuación a medida que aumenta la edad. También las educadoras expresan esta relación inversa entre edad y conductas externalizantes e internalizantes, mostrando que a mayor edad menor nivel de conductas problema. En general, los estudios muestran que las conductas internalizantes aumentan a medida que los niños van creciendo, lo contrario de lo observado en nuestro estudio (Arias et al., 2009; López-Soler et al., 2010), pudiendo deberse al grupo etáreo elegido, 2 a 5 años, donde aparecen más comportamientos difíciles relacionados con lo que se ha descrito como la fase del primer oposicionismo. La escasa producción científica sobre niños y niñas de 2 a 5 años en este tipo de conductas dificulta su comparación.

Padres y educadoras coinciden al constatar que a menor NSE mayor nivel de conductas externalizantes e internalizantes, resultados que coinciden con trabajos precedentes (Jaramillo, 2015). El nivel socioeconómioco guarda relación con los estilos parentales, por lo que la relación está mediada por el tipo de prácticas parentales con el que se educa. Por consiguiente, el NSE es una variable mediadora del estilo parental (Contreras, 2016). Esto estilos educativos como han sido denominados por Murillo et al. (2015) son en definitiva prácticas socializantes para niños/niñas en la infancia.

En relación al tercer objetivo, prácticas parentales positivas, inconsistentes y castigadoras y su relación con conductas externalizantes e internalizantes en función del sexo, edad y NSE, los resultados del presente trabajo coinciden con el de Contreras (2016) en el que la relación entre NSE alto y PP/positivas no se confirma, pero sí con PP/negativas (Gubbins, 2014). Los estudios inciden en que, en hogares con características adaptativas, interés en la educación y vínculos seguros se utilizan prácticas parentales positivas en la educación (García et al., 2014), pero que, en hogares disruptivos, marcados por la violencia o con niveles económicos muy desfavorecidos, son más frecuentes las prácticas parentales castigadoras (Bolívar et al., 2014).

Los resultados sobre la relación entre prácticas parentales y sexo también aparecen en investigaciones previas, mostrando que los padres tienden a percibir más conductas desadaptativas en los niños que en las niñas ya preescolares (Bolivar, 2007). De hecho, el empleo de prácticas castigadoras de los padres es más frecuente con niños y niñas de 4 que de 2 años. También es importante considerar los cambios evolutivos. Niños y niñas van adquiriendo mayor autonomía progresivamente y son capaces de un repertorio más amplio de expresiones de conductas de desobediencia, hostilidad o desafío, lo que 
puede favorecer, en padres sin recursos alternativos, el empleo de prácticas punitivas. El castigo físico es una práctica muy recurrente, muchas veces invisibilizado, tolerado y validado en Latinoamérica (Isaza y Henao, 2012).

El tipo de prácticas parentales más frecuente se relacionó con el NSE, en concreto, las prácticas parentales negativas. Se observó menor frecuencia de prácticas inconsistentes y castigadoras a mayor NSE. Similares resultados se hallaron en otros estudios (Cabrera et al., 2012; Franco et al., 2014; Gómez et al., 2014) encontrando que los padres de niveles medio y alto tendrían prácticas más adecuadas que los padres de NSE bajo, quienes tenían una peor percepción de los niños/niñas y generaban conductas más rudas con sus hijos/hijas. El informe de las educadoras presenta resultados coincidente con los pares, por lo que los ambientes desfavorecidos cobran gran importancia en la explicación de conductas desadaptativas (Contreras, 2016). En este trabajo queda de manifiesto la relación entre prácticas parentales y conductas desadaptativas en niños/niñas, y las diferencias de género en la percepción de dichas conductas entre padres y educadoras. La relevancia de estos resultados nos obliga a pronunciarnos a favor programas de intervención parental que apunten a las poblaciones en mayor riesgo social, potenciando las figuras parentales con el fin de mejorar los índices de salud mental en los niños.

\section{REFERENCIAS}

Achenbach, T.M., y Rescorla, L.A. (2010). Manual for the ASEBA Preschool Forms and Profiles. Burlington, VT: University of Vermont, Research Center for Children, Youth, and Families.

Arias, G., Montoya, E. y Romero, M. (2009). Manifestaciones de Conducta Disruptiva y Comportamiento Perturbador en Población Normal de 4 A 17 Años de Edad. AGO.USB Medellin-Colombia, 9(1), 17-33.

Bolívar, L., Convers, A., y Moreno, J. (2014). Factores de riesgo psicosocial asociados al maltrato infantil. Psychologia: avances de la disciplina, 8, 67-76.

Bellei, C. (2013) El estudio de la segregación socioeconómica y académica de la educación chilena. Estudios. Pedagógicos, 39, 325-345.

Cabrera, V., González, M., y Guevara, I. (2012) Estrés parental, trato rudo y monitoreo como factores asociados a la conducta agresiva. Univ. Psychology, 11, 241-254.

Chaplin, T. (2015). Gender and emotion expression: a developmental contextual perspective. Emotion Review, 7, 14-21.

Clerkin, S., Marks, D., Policaro, K., y Halperin, J. (2007). Psychometric properties of the Alabama Parenting Questionnaire Preschool Revision. Journal of Clinical Child and Adolescent Psychology, 36, 19-28.

Contreras, G. (2016). Prácticas parentales y su relación con conductas externalizadas e internalizadas de niños y niñas en preescolar. Tesis Doctoral. San Sebastián: Universidad del País Vasco.

Cova, F., Maganto, C., y Melipillán, R. (2005). Adversidad familiar y desarrollo de conductas externalizadas e internalizadas en preadolescentes. Revista Chilena de Neuro-Psiquiatría, 43, 287-296. 
Darling, N., y Steinberg, L. (1993). Parenting style as context: an integrative model. Psychological Bulletin, 113(3), 487-496.

Franco, N., Pérez, M., y de Dios, M. (2014). Relación entre los estilos de crianza parental y el desarrollo de ansiedad y conductas disruptivas en niños de 3 a 6 años. Revista de Psicología Clínica con Niños y Adolescentes, 1, 149-156.

García, M., Rivera, S., y Reyes, I. (2014). La precepción de los padres sobre la crianza de los niños. Acta Colombiana de Psicología, 17, 133-141.

Gómez, A., Santelices, M., Gómez, D., Rivera, C., y Farkas, Ch. (2014). Problemas conductuales en preescolares chilenos: percepción de las madres y del personal educativo. Estudios Pedagógicos, 40, 175-187.

Gubbins, V. (2014). Estrategias educativas de familias de clase alta, un estudio exploratorio. Revista Mexicana de Investigación Educativa, 19, 1069-1089.

Hewitt, N., y Moreno, J. (2013). Programa Entre Tres: estudio piloto de un programa de intervención psicológica para el manejo de comportamientos internalizados $y$ externalizados. Grupo de investigación Avances en Psicología Clínica y de la Salud. Bogota: Universidad de San Buenaventura.

Isaza, L., y Henao, G. (2012). Influencia del clima sociofamiliar y estilos de interacción parental sobre el desarrollo de habilidades sociales en niños y niñas. Persona, 15, 253-271.

Jaramillo, J. (2015). "Este es chorro": identificaciones masculinas y aprendizajes infantiles en contextos urbanos de Argentina. Antípoda Revista Antropológica Arqueológica, 23, 113-134.

Lecannelier, F., Pérez, C., Groissman, S., Gallardo, D., Bardet, A., Bascuñan, A., y Rodríguez, J. (2014). Validación del Inventario de Conductas Infantiles para niños de entre 11/2-5 años (CBCL 11/2-5) en la Ciudad de Santiago. Universitas Psychologica-Bogotá Colombia, 13, $15-24$.

López-Soler, C., Alcántara, M., Fernández, V., Castro, M., y López, J. (2010). Características y prevalencia de los problemas de ansiedad, depresión y quejas somáticas en una muestra clínica infantil de 8 a 10 años, mediante el CBCL. Anales de Psicología, 26, 325-334.

Maganto, C., y Garaigordobil, M. (2010). Estudio psicométrico del Screening de Problemas de Conducta Infantil SPCI. Psicothema, 22, 316-322.

Moreno, J., Echavarría, K., Pardo, A., y Quiñones, Y. (2014). Funcionalidad familiar, conductas internalizadas y rendimiento académico en un grupo de adolescentes de la ciudad de Bogotá. Psychologia: avances de la disciplina, 8, 37- 46.

Murillo, A., Priegue, D., y Cambeiro, M. (2015). Una aproximación a los estilos educativos como prácticas socializadoras. Revista de Estudios e Investigación en Psicología y Educación, Extra 5.

Tani, F., y Smorti, M. (2013). Romantic relationships in adolescents with internalizing and externalizing problems. . European Journal of Child development, Education and Psychopathology, 1(1), 31-44.

Van der Voort, A., Linting, M., Juffer, F., Bakermans-Kranenburg, M., Schoenmaker, C., y van Ijzendoorn, M. (2014). The development of adolescent's internalizing behavior: longitudinal effects of maternal sensitivity and child inhibition. Journal Youth Adolescence, 43, 528-540.

Recibido: 25 de julio de 2018

Recepción Modificaciones: 12 de septiembre de 2018

Aceptado: 15 de noviembre de 2018 